\title{
PNPLA3 gene polymorphism and severity of liver steatosis and fibrosis
}

Marta Wawrzynowicz-Syczewska ${ }^{1}$, Aleksandra Waszczyk ${ }^{1}$, Dorota Bander ${ }^{1}$, Janusz Miętkiewski Anna Urbańska³, Kaja Scheibe ${ }^{3}$

\author{
${ }^{1}$ Department of Infectious Diseases, Hepatology and Liver Transplantation, \\ Pomeranian Medical University, Szczecin, Poland \\ ${ }^{2}$ Department of Pathology, Marie-Curie Hospital, Szczecin, Poland \\ ${ }^{3}$ Department of Infectious, Tropical Diseases and Immune Deficiency, \\ Pomeranian Medical University, Szczecin, Poland
}

Submitted: 12 October 2020

Accepted: 7 February 2021

Arch Med Sci Civil Dis 2021; 6: e31-e35

DOI: https://doi.org/10.5114/amscd.2021.105522

Copyright @ 2021 Termedia \& Banach

\section{Abstract}

Introduction: Non-alcoholic fatty liver disease (NAFLD) is one of the leading chronic liver diseases worldwide. Environmental and genetic factors have an impact on NAFLD development. Single nucleotide polymorphism in the PNPLA3 gene is strongly related to the increased fat content in hepatocytes. The aim of our study was to investigate the association between PNPLA3 variants and the intensity of liver steatosis and fibrosis assessed by liver biopsy in patients with NAFLD.

Material and methods: The study group comprised 127 patients with NAFLD confirmed by liver biopsy. The control group comprised 251 healthy volunteers. In every case genotyping of the PNPLA3 rs738409 C to G nonsynonymous sequence variant was performed. Distributions of genotypes and alleles were compared between NAFLD patients and controls, and analyzed in relation to different steatosis and fibrosis stages in the study group.

Results: Frequencies of PNPLA3 genotypes strongly differ between NAFLD patients and controls $(p=0.00001)$. Odds ratio $(\mathrm{OR})$ for genotype GG in NAFLD was $2.94(1.45-5.97, p=0.0015)$. Also allele $\mathrm{G}$ was more frequent in NAFLD patients vs. controls $(p=0.00001)$; OR for $\mathrm{G}$ allele in NAFLD was 2.74 (1.77-4.27, $p=0.00000273$ ). No significant differences in the genotype or allele distribution of PNLPA3 were observed in relation to steatosis intensity or between patients with or without cirrhosis. However, allele G was found is every hepatocellular carcinoma case.

Conclusions: We do not advocate universal genetic testing in every NAFLD case to select patients at risk of development of cirrhosis or steatohepatitis. Further investigation of the relationship between PNPLA3 variants and hepatocellular carcinoma is warranted.

Key words: fibrosis, steatosis, non-alcoholic fatty liver disease, patatin-like gene polymorphism.

\section{Introduction}

Over the last decades, a noticeable increase in the incidence and prevalence of fatty liver disease unrelated to alcohol consumption has been observed worldwide [1]. Non-alcoholic fatty liver disease (NAFLD) encompasses two types: simple liver steatosis (NAFL) with or without mild lobular inflammation, and non-alcoholic steatohepatitis

\author{
Corresponding author: \\ Marta Wawrzynowicz- \\ Syczewska \\ Department of \\ Infectious Diseases, \\ Hepatology and \\ Liver Transplantation \\ Pomeranian Medical \\ University \\ Szczecin, Poland \\ Phone: +48 501179005 \\ E-mail: martaws@wp.pl
}


(NASH), which is characterized by various degrees of steatosis, necroinflammatory changes, ballooning, and fibrosis [2]. NAFLD can progress to cirrhosis and hepatocellular carcinoma (HCC) and is a significant indication for liver transplantation.

NAFLD prevalence is estimated at $25.2 \%$, with the highest prevalence in the Middle East and South America [3]. The increasing incidence of NAFLD is closely associated with the alarming rates of obesity and diabetes. Other risk factors for NAFLD include insulin resistance, hypertension, hyperlipidemia, race, and ethnicity. There is also evidence of familial presentation, suggesting that genetic and environmental factors influence NAFLD development. Recent genome-wide association studies have identified a single variant in the patatin-like phospholipase domain-containing 3 gene (PNPLA3) encoding a triacylglycerol lipase [4]. Mutations in the rs738409 region, resulting in a cytosine-to guanine-substitution, change codon 148 from isoleucine to methionine (I148M). This results in the loss of lipase activity, as the PNPLA3-/148M allele $(G)$ appears to be a major determinant of increased fat content in hepatocytes [5]. The G allele is also proposed to be associated with disease progression. The PNPLA3-I148M allele frequency is consistent with NAFLD prevalence in different ethnic groups [5].

Herein, we investigated the association between the PNPLA3 rs738409 polymorphism and liver steatosis and fibrosis in a cohort of $127 \mathrm{pa}$ tients diagnosed with NAFLD based on liver biopsy. Special attention has been paid to the relationship between the p.I148M variant of PNPLA3 and the intensity of steatosis and fibrosis.

\section{Material and methods}

\section{Study population}

The study population comprised 127 Polish patients with chronic liver disease diagnosed with NAFLD based on steatotic echo imaging of the liver by ultrasound, elevated liver enzymes, and the exclusion of known viral and non-viral liver diseases. In every case, substantial alcohol intake ( $\leq 20 \mathrm{~g}$ for women, $\leq 30 \mathrm{~g}$ for men daily) was ruled out according to the patient's statement and no observations of alcohol-associated laboratory abnormalities, such as aspartate aminotransferase activity elevated more than 1.5-fold higher than alanine aminotransferase (ALT) activity, significantly elevated $\gamma$-glutamyltransferase, elevated bilirubin, and a high mean corpuscular volume of red blood cells or increased carbohydrate-deficient transferrin. Steatosis was confirmed by liver biopsy performed on all study participants.
Steatosis was graded according to the following NAFLD activity scores [6]: grade 0 , no steatosis (< $5 \%$ of hepatocytes with fat droplets); grade 1 , mild steatosis ( $5-<30 \%$ of steatotic hepatocytes); grade 2 , moderate steatosis ( $\leq 30 \%-$ $<60 \%$ of steatotic hepatocytes); and grade 3 , severe steatosis ( $\leq 60 \%$ of steatotic hepatocytes). Patients with no steatosis were excluded. NASH was diagnosed when steatosis was associated with lobular inflammation, hepatocyte ballooning, and a variable degree of fibrosis [2]. Fibrosis was assessed according to the 5-stage METAVIR grading and staging scale, where stage 0 indicates no fibrosis and stage 4 is consistent with liver cirrhosis. According to the fibrosis stage, patients were divided into two groups: non-cirrhotic and cirrhotic.

The control group comprised 251 healthy volunteers who displayed no steatosis in the ultrasound and no elevations in liver tests.

\section{Genotyping assay}

The Blood/Cell DNA Mini Kit (Syngen, Wrocław, Poland) was used to isolate genomic DNA from EDTA-anticoagulated blood. The PNPLA3 rs738409 $C$ to $G$ nonsynonymous sequence variant was genotyped in all subjects and controls using the TaqMan SNP Genotyping Assay (Thermo Fisher Scientific, Waltham, MA, USA) according to the manufacturer's protocol. Genotyping of each sample was analyzed automatically using Step One Software v.2.2.2.

\section{Ethics approval}

The approval of the Bioethics Committee was obtained from the Review Board of the Pomeranian Medical University in Szczecin (KB001/104/17). All study participants gave their written consent. The participants were assured of the right to withdraw their consent at any time with any precondition.

\section{Statistical analysis}

Statistica 13 software (StatSoft, Krakow, Poland) was used for analyses. Data were presented as medians and ranges and were analyzed using Student's $t$-test and the $\chi^{2}$ test for parametric and nonparametric variables, respectively. Differences in the frequencies of the investigated genetic variants of PNPLA3 in the study and control groups were assessed by $\chi^{2}$ test, and the odds ratios with $95 \%$ confidence intervals $(\mathrm{OR}, 95 \% \mathrm{Cl})$ for homozygous mutant genotypes and risk alleles were calculated. Differences in genotype frequencies were analyzed in relation to the degree of steatosis, NASH, or advanced liver fibrosis. 


\section{Results}

Altogether, 127 patients with NAFLD assessed by liver biopsy were prospectively recruited between January 2018 and June 2019. There were 76 (60\%) female and 51 (40\%) male patients with a median age of 58 years (range: $23-75$ years) and a median body mass index (BMI) of $31.4 \mathrm{~kg} / \mathrm{m}^{2}$ (range: $23.3-49.5 \mathrm{~kg} / \mathrm{m}^{2}$ ) in the study group. The control group comprised 251 healthy volunteers, 135 (54\%) females and 116 (46\%) males, with a median age of 37 years (range: 19-84 years) and a median BMI of $25.0 \mathrm{~kg} / \mathrm{m}^{2}$ (range: $16-41.7$ $\mathrm{kg} / \mathrm{m}^{2}$ ). These two groups (Table I) did not differ significantly with respect to sex distribution ( $p=$ $0.26)$, but the controls were significantly younger $(p<0.001)$ and had lower BMIs $(p<0.001)$.

Genetic testing was performed for all 378 study participants. In the whole cohort, 343 and 173 subjects carried alleles C (91\%) and G (46\%), respectively. Genotype frequencies were as follows: CC, 205 (54\%); CG, 138 (37\%); and GG, 35 (9\%). The frequencies were consistent with Hardy-Weinberg equilibrium. Genotype CC was present in 48 (38\%) patients and 157 (63\%) controls, genotype CG was found in 48 (46\%) NAFLD patients and 79 (31\%) controls, and the mutant genotype GG was present in 20 (16\%) patients and $15(6 \%)$ controls (Table I). Therefore, the distribution of PNPLA3 genotypes strongly differed between NAFLD patients and healthy controls ( $p=0.00001$ ). The odds ratio for genotype GG in patients vs. controls was $2.94(1.45-5.97, p=0.0015)$. Allele $\mathrm{G}$ was more frequent in patients with NAFLD: 79 $(62 \%)$ vs. $94(37 \%)$ in controls, OR $=2.74$ (1.774.27), $p=0.00001$ and 0.00000273 , respectively.

Mild, moderate, and severe liver steatosis was found in 47 (37\%), 53 (41.7\%), and 27 (21.3\%) patients, respectively. There were no differences in age, sex, BMI, and ALT activity between these patients (Table I). Neither PNPLA3 genotypes nor alleles showed significant distribution differences in relation to the degree of steatosis (Table I). Advanced fibrosis consistent with liver cirrhosis was diagnosed in 22 (17.3\%) patients, and NASH in $15(12 \%)$ patients. There were no statistically significant differences in the PNPLA3 genotype distribution between cirrhotic and non-cirrhotic patients. However, the $\mathrm{C}$ allele was significantly more frequent in non-cirrhotic patients than in cirrhotic patients $(p=0.023)$. The odds ratio for cirrhosis in $\mathrm{G}$ allele carriers was 1.78 (0.64-4.91), which was similar to that of non-cirrhotic patients $(16 / 22$ vs. $63 / 105, p=0.14)$. There were no differences in genotype distributions between $\mathrm{NASH}$ and non-NASH patients (data not shown). Notably, among 7 patients with HCC, six were G allele carriers and five possessed homozygous GG mutant genotype.

\section{Discussion}

Our study confirms that the PNPLA3 I148M allele is associated with liver steatosis, as CG and GG genotypes were more frequent in patients with NAFLD than in healthy controls. This result is consistent with those from other studies [7, 8]. However, we observed no significant relationship between the PNPLA3 risk allele and the degree of fat content determined using liver biopsy, serum ALT activity, or NAFLD severity indicated by advanced fibrosis or necroinflammatory activity. These latter observations are in contrast to those from other studies showing that the $G$ allele influenced the susceptibility and histological severity of NAFLD [7, 9]. This discrepancy may be due to the small cirrhosis/NASH cohort size in our study. However, there was no difference in the risk allele distribution in relation to ALT, which is another marker of liver injury. The $G$ allele also showed no effect on serum ALT activity in a pediatric cohort [8]. In our study, however, non-cirrhotic patients more frequently carried the wild-type $C$ allele of PNPLA3 (Table I). We hypothesize that the $\mathrm{C}$ allele may confer a protective effect against fibrosis progression. Interestingly, in cirrhotic patients with HCC, the homozygous mutant genotype GG was detected in five of seven HCC patients, and another patient was a CG genotype carrier. This potential association with HCC warrants further study.

The limitations of our study include differences in age and BMI between NAFLD patients and controls as well as having few patients with NASH/ cirrhosis. However, our study investigated PNPLA3 genotypes and allele frequencies in relation to the steatosis degree assessed by liver biopsy.

In conclusion, we confirmed a strong relationship between liver steatosis and the p./148M PNPLA3 allele in the Polish cohort. As we were unable to confirm the influence of the p.I148M risk variant on liver disease progression or NASH development, we do not advocate universal genetic testing in every NAFLD case. However, in patients with advanced liver disease, determination of the rs738409 polymorphism may help identify individuals at risk of HCC, which should be confirmed in further studies.

\section{Acknowledgments}

This work was supported by a grant from the Pomeranian Medical University, Szczecin, Poland (grant number WLA-162-02/S/17/2018).

\section{Conflict of interest}

The authors declare no conflict of interest. 


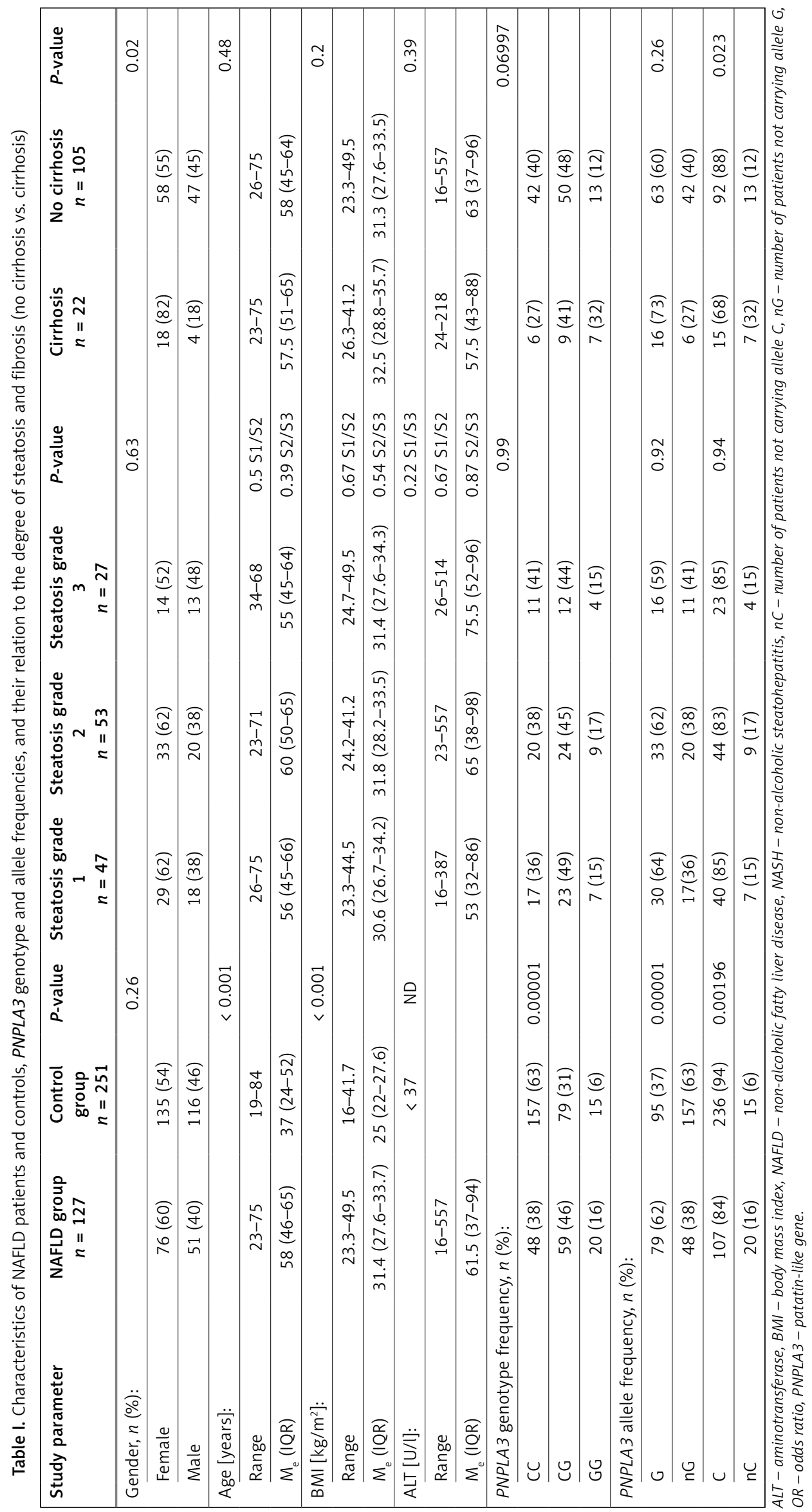




\section{References}

1. Asrani SK, Devarbhavi H, Eaton J, Kamath PS. Burden of liver disease in the world. J Hepatol 2019; 70: 151-71.

2. Chalasani N, Younossi Z, Lavine JE, et al. The diagnosis and management of non-alcoholic fatty liver disease: practice guideline by the American Association for the Study of Liver Disease, and the American Gastroenterological Association. Hepatology 2012; 55: 2005-23.

3. Younossi ZM, Koenig AB, Abdelatif D, Fazel Y, Henry L, Wymer M. Global epidemiology of nonalcoholic fatty liver disease-Meta-analytic assessment of prevalence, incidence, and outcomes. Hepatology 2016; 64: 73-84.

4. Karlsen TH, Melum E, Franke A. The utility of genome-wide association studies in hepatology. Hepatology 2010; 55: 1833-42.

5. Romeo S, Kozlitina J, Xing C, et al. Genetic variation in PNPLA3 confers susceptibility to nonalcoholic fatty liver disease. Nat Genet 2008; 40: 1461-5.

6. Brunt EM, Kleiner D, Wilson LA, Belt P; Neuschwander-Tetri BA for the NASH Clinical Research Network. Nonalcoholic fatty liver disease (NAFLD) activity score and the histopathologic diagnosis in NAFLD: distinct clinicopathologic meanings. Hepatology 2011; 53: 810-20.

7. Dai G, Liu P, Li X, Zhou X, He S. Association between PNPLA3 rs738409 polymorphism and nonalcoholic fatty liver disease (NAFLD) susceptibility and severity. Medicine 2019; 98: e14324.

8. Krawczyk M, Liebe R, Maier IB, Engstler AJ, Lammert F, Bergheim I. The frequent adiponutrin (PNPLA3) variant p.lle148Met is associated with early liver injury: analysis of a German pediatric cohort. Gastroenterol Res Pract 2015; 2015: 205079.

9. Sookoian S, Pirola CJ. Meta-analysis of the influence of I148M variant of patatin-like phospholipase domain containing 3 gene (PNPLA3) on the susceptibility and histological severity of nonalcoholic fatty liver disease. Hepatology 2011; 53: 1883-94. 